\title{
Impact of Channel Models on the End-to-End Performance of mmWave Cellular Networks
}

\author{
Michele Polese, Michele Zorzi \\ Department of Information Engineering, University of Padova, Italy \\ e-mail: \{polesemi, zorzi\}@ dei.unipd.it
}

\begin{abstract}
Communication at mmWave frequencies is one of the major innovations of the fifth generation of cellular networks, because of the potential multi-gigabit data rate given by the large amounts of available bandwidth. The mmWave channel, however, makes reliable communications particularly challenging, given the harsh propagation environment and the sensitivity to blockage. Therefore, proper modeling of the mmWave channel is fundamental for accurate results in system simulations of mmWave cellular networks. Nonetheless, complex models, such as the 3GPP channel model for frequencies above $6 \mathrm{GHz}$, may introduce a significant overhead in terms of computational complexity. In this paper we investigate the trade offs related to the accuracy and the simplicity of the channel model in end-to-end network simulations, and the impact on the performance evaluation of transport protocols.

Index Terms-Channel model, mmWave, simulation
\end{abstract}

\section{INTRODUCTION}

The next generation of cellular networks (5G) targets massive improvements in several Key Performance Indicators (KPIs) to sustain the mobile traffic growth and the new use cases that will emerge in the near future. In particular, 5G networks will support very high throughput, combined with ultra-low latency, and high reliability and device density [1], [2]. MmWave communications, together with the enhancements in spectral efficiency and network densification, are a possible enabler for the multi-gigabit-per-second data rates envisioned in future $5 \mathrm{G}$ networks [3]. Thanks to the wide availability of free spectrum at such high frequencies, network operators can allocate a much larger bandwidth with respect to the sub- $6 \mathrm{GHz}$ frequencies traditionally used for cellular communications.

The mmWave bands, however, are characterized by a harsh propagation environment that makes it difficult to reliably deploy a truly mobile mmWave network. The two main issues are the propagation loss, which is proportional to the square of the carrier frequency, and the blockage caused by common materials such as brick, mortar and also the human body [4]. The first challenge can be addressed using directional antennas. Thanks to the smaller wavelengths, the same area can be packed with more antenna elements at mmWave frequencies than in the sub- $6 \mathrm{GHz}$ band, and, therefore, it is possible to increase the link budget with beamforming techniques [5]. On the other hand, an ultra-dense deployment can help to avoid blockage phenomena and reduce the outage probability [6].

New challenges related to the nature of the mmWave spectrum emerge also throughout the whole protocol stack. For example, the sudden transition between Line of Sight (LOS)

This work was partially supported by the U.S. Department of Commerce/NIST (Award No. 70NANB17H166) and by the CloudVeneto initiative. and Non Line of Sight (NLOS) states and the consequent drop in the channel quality may cause latency and efficiency issues to end-to-end data flows relying on the Transmission Control Protocol (TCP) [7], [8], and the need for frequent and fast beam adaptation and/or handover calls for the design of efficient mobility management procedures [9]. Given the impact of mmWaves on the full stack, it is important to design and evaluate algorithms and protocols considering the performance of end-to-end systems. In this regard, network simulators are a valuable tool, whose reliability for wireless simulation, however, largely depends on the accuracy of the channel model [10].

In general, system level simulators consider a packet as a basic simulation unit, and do not model the actual bit transmission on the wireless link. The latter is usually abstracted with an error model, which maps the link Signal to Interference plus Noise Ratio (SINR) on packet error probability curves to decide if the packet transmission was successful or not [11]. Therefore, the correct modeling of the transmission dynamics depends on the accuracy of the model for channel propagation and fading. In particular, given the characteristics of mmWave frequencies, the combined effect of propagation, fading and beamforming has a much higher impact on the end-to-end performance than in the sub-6 GHz band.

The publicly available ns-3 mmWave module [12], that simulates $5 \mathrm{G}$ cellular networks at mmWave frequencies, features the implementation of the 3GPP channel model for frequencies above $6 \mathrm{GHz}$ [13], [14]. This is the channel model that, according to $3 \mathrm{GPP}$, should be used for the simulations involving its latest standard, NR, that also supports mmWave frequencies. However, it is a very complex model, which requires the generation of a large number of random numbers throughout the simulation, therefore limiting the scalability of the simulated scenarios. On the other hand, simpler channel models have been used in analytical studies in the literature, based on an abstraction of the beamforming gain and on Rayleigh or Nakagami fading [15].

In this paper we compare the effects of the 3GPP channel model, which is used as a reference, and of a Nakagami fading-based channel model on the end-to-end performance of a mmWave cellular network. We show that there exists a trade off between the accuracy, defined as the difference in the considered metrics with respect to the reference $3 \mathrm{GPP}$ model, and the scalability of the simulations. In particular, the Nakagami-fading-based model introduces more severe fading than the 3GPP model, and, consequently, in the evaluated scenarios, always yields a lower throughput, but it reduces the 
simulation execution time by at least an order of magnitude.

The remainder of the paper is organized as follows. In Sec. II we describe the main features of the 3GPP channel model, and review the models used in analytical studies. Then, in Sec. III we introduce the implementation of the channel model which will be compared against the 3GPP one, and present the results of the comparison in Sec. IV] Finally, in Sec. V] we conclude the paper and address possible avenues of future work.

\section{OVERVIEW OF MMWAVE CHANNEL MODELS}

In recent years, there have been several channel measurement campaigns at mmWave frequencies to characterize their propagation and fading, in different environments and conditions [16], [17]. The main characteristics of the mmWave channel can be summarized as (i) a clear difference between LOS and NLOS propagation; (ii) higher penetration loss than at sub-6 GHz frequencies; (iii) sparsity in the angular domain; and (iv) reduced impact of small scale fading [15]. Several channel models, which capture the nature of mmWave propagation, have also been proposed, and a review of the main contributions can be found in [18].

The modeling usually comprises a propagation loss model and a fading model. The propagation loss is computed by assigning to each physical location in the scenario a LOS probability (unless deterministic environment models are used), and by applying different equations according to the LOS or NLOS state of the user. A survey on path loss models can be found in [19]. For fading, popular measurement-based channel models at mmWave frequencies [6], [13], [20], [21] are extensions of the WINNER and WINNER-II Spatial Channel Models (SCMs) [22], while analytical studies generally use Rayleigh or Nakagami fading [23].

The 3GPP channel model for mmWave frequencies, which will be used as the reference model in this paper, has been standardized in [13]. This SCM is based on a channel matrix $\mathbf{H}$, whose entry $(i, j)$ represents the channel between the $i$ th and the $j$-th antenna elements at the transmitter and the receiver, respectively, and depends on the combined effect of $N$ multiple paths, i.e., the clusters. The clusters represent the direct LOS path (if present) and the reflections that contribute to the total received power. Each of them is modeled using a different delay and power, and is composed by multiple rays, distributed around a common cluster angle of arrival and departure. Moreover, two different components contribute to the modeling of fading [13]: (i) large scale parameters, which are based on the user mobility and/or updates in the scenario, and affect the shadow fading, the delay spread of the clusters, the angular spread of the rays, and, in LOS, the Ricean factor 1 associated to the LOS cluster; and (ii) fast fading components, that model the small scale variations related to each cluster's delay and power, the actual angle of arrival and departure of the rays and the Doppler spread. The 3GPP channel model, and SCMs in general, can be easily integrated in the simulation with realistic beamforming, given that the beamforming gain can be

\footnotetext{
${ }^{1}$ The term Ricean factor is used in [13] to refer to the relative strength of the direct path with respect to the scattered components of a LOS channel, for any fading model (not necessarily Ricean).
}

computed by directly applying the beamforming vectors at the transmitter and receiver to the channel matrix $\mathbf{H}$ [6].

The parameters of the 3GPP channel are random variables generated from specific distributions, as detailed in [13]. The total number of random variables drawn and, in general, of computations for each transmitter/receiver pair in the scenario is proportional to $U \times S \times N$, with $U$ and $S$ the total number of antenna elements at the transmitter and the receiver. This can significantly increase the complexity of system level simulations, especially at mmWave frequencies, where antenna arrays with many elements are used to make up for the high propagation loss. In particular, random variables related to fast fading parameters are drawn at each transmission, while large scale fading parameters can be updated periodically2. Avoiding the update of the large scale fading parameters at each transmission is a practical assumption that helps decrease the computational complexity of the 3GPP channel model. However, despite the important efforts related to channel modeling at mmWave frequencies, there is a lack of statistical models of the rate at which large scale fading parameters evolve at mmWave frequencies, and only recently have results been presented in the case of specific blockage events [24]. Therefore, in the ns-3 implementation [14], it is possible to configure different large scale fading parameter update intervals.

Despite their accuracy and natural relation with beamforming techniques [10], SCMs cannot be used for analytical studies. Therefore, papers that investigate mmWave network performance analytically have proposed other channel abstractions, which use similar propagation loss equations, but simplify the modeling of fading and beamforming. For example, in [23], the authors derive results on the coverage and rate of mmWave cellular networks using a channel model based on different path loss laws for LOS and NLOS, Nakagami fading and a simplified sectored beamforming. Nakagami fading, introduced in [25], depends on a parameter $m$ which controls the amplitude of the fading phenomena: the larger $m$, the less severe the variations in amplitude. The sectored beamforming model computes the beamforming gain $G$ by dividing the angular space in two regions: the main lobe, of angular width $\theta_{b}$ and maximum gain $G_{M}$, and the complementary sector with gain $G_{m}$. The total beamforming gain is given by the product of the transmitter and receiver gains, which can be either $G_{M}$ or $G_{m}$ according to the mutual position of the transmitter and the receiver. Similar approaches can be found in [15], [26], [27]. Other papers use Rayleigh fading for tractability, because it provides a lower bound to the system performance with respect to Nakagami fading in a stochastic geometry analysis [28]. Nakagami fading, however, is generally preferred because it models more realistically the impact of fast fading on mmWave links [15], and returns Rayleigh fading for $m=1$. These channel models are usually computationally very efficient, because the number of random variables to be generated for each transmitter/receiver pair does not depend on the number

\footnotetext{
${ }^{2}$ The report [13] also introduces a spatially consistent procedure for the computation of the large scale fading parameters, which generates random numbers correlated with those drawn at the previous update based on the distance the user has covered between two consecutive updates.
} 
of antenna elements and channel clusters.

To the best of our knowledge, SCMs and Nakagami-based models have not been compared for the evaluation of the performance of an end-to-end mmWave network. In [29], the authors compare different SCMs for mmWave cellular networks, but they only consider link level metrics for their evaluation. Similarly, [30] compares the different models available in the ns-3 mmWave module using as performance metric the Medium Access Control (MAC) layer throughput of a single user. In this paper, we propose the implementation of a simple channel model, based on Nakagami fading, and compare it with the 3GPP channel model, focusing on the trade off between the accuracy of the simulation results and the computational complexity.

\section{SySTEM MODEL}

The ns-3 mmWave module [12] can be used to simulate endto-end mmWave networks, with realistic deployments, modeling of obstacles, a complete protocol stack for the Radio Access Network (RAN), a simple model for the core network and a full implementation of the TCP/IP stack. The physical layer in the base stations and User Equipments (UEs) is based on Orthogonal Frequency Division Multiplexing (OFDM), and features a flexible frame structure [31], that can be adapted to simulate different $5 \mathrm{G}$ use cases [1], with a dynamic Time Division Duplexing (TDD) scheduling mechanism that adapts the duration of the scheduling intervals to the amount of data to be transmitted. It is also possible to simulate users' mobility and dual connectivity with Long Term Evolution (LTE) networks [9].

The module is equipped with different channel models [12]: there are two SCMs, i.e., the 3GPP [14] and the NYU channel models [6], and the possibility of using ray-tracing or measured channel traces. In addition to the available models, in this paper we introduce the MmWaveSimpleChannel class, which implements a channel model based on Nakagami fading and simplified beamforming, inspired to the widely-used models for mmWave cellular networks analysis in [15], [23], [26], [27].

The SINR for the link between the transmitter $i$ and the receiver $j$ is

$$
P_{r x, j}=\frac{P_{t x, i} h_{i, j} G_{i, j} L_{i, j}}{\sigma^{2}+\sum_{k \in \mathcal{I}} P_{t x, k} h_{k, j} G_{k, j} L_{k, j}},
$$

where $P_{t x, i}$ is the transmission power, $h_{i, j}$ the Nakagami fading, $G_{i, j}$ the beamforming gain and $L_{i, j}$ the pathloss for link $i, j$. The set $\mathcal{I}$ contains all the devices which are interfering with the transmission on link $i, j$, i.e., those that are actively transmitting during the same time interval and in the same frequency band as $i$ and $j$. Given that the modeling of the pathloss is not as computationally intensive as that of the fading, we use the 3GPP model for the propagation loss $L_{i, j}$, which depends on the LOS condition, the 2D and 3D distance and the height of the devices, and the deployment scenario considered. Moreover, it is possible to select whether to enable or not the correlated shadowing [13]. Different $m$ values of the Nakagami fading for LOS and NLOS links, respectively $m_{\mathrm{LOS}}$ and $m_{\mathrm{NLOS}}$, can be selected when configuring the simulation. We will provide insights on the choice of $m$ in the next section.
Beamforming is modeled by computing the gain for Uniform Planar Arrays (UPAs) with isotropic elements. In our future work, we plan to relax this modeling assumption and account for more realistic antenna patterns. Following the approach described in [32], [33], we compute the array radiation pattern $A_{A, i}\left(\theta^{i, j}, \phi^{i, j}, \theta_{s}^{i}, \phi_{s}^{i}\right)$ for device $i$ with respect to the signal to/from device $j . \theta^{i, j}$ and $\phi^{i, j}$ are the vertical and horizontal angles associated to the LOS direction between $i$ and $j$, and $\theta_{s}^{i}$ and $\phi_{s}^{i}$ are the vertical and horizontal steering angles for $i$. Since the antenna elements are isotropic, the array radiation pattern for an antenna array of $n$ elements is equal to the array factor (in dB) [33]:

$$
\begin{aligned}
A_{F, i}\left(\theta, \phi, \theta_{s}, \phi_{s}\right) & =10 \log _{10}\left[1+\rho\left(\left|\mathbf{a}(\theta, \phi) \mathbf{w}^{T}\left(\theta_{s}, \phi_{s}\right)\right|^{2}-1\right)\right] \\
& \stackrel{\rho=1}{=} 10 \log _{10}\left(\left|\mathbf{a}(\theta, \phi) \mathbf{w}^{T}\left(\theta_{s}, \phi_{s}\right)\right|^{2}\right),
\end{aligned}
$$

where we consider $\rho=1$ and omit the dependency on $i$ and $j$ in the angles. $\mathbf{a} \in \mathbb{C}^{n}$ represents the phase shift due to the placement of the antenna elements [32], i.e.,

$$
\begin{aligned}
\mathbf{a}(\theta, \phi) & =\left[a_{1,1}, a_{1,2}, \ldots, a_{1, \sqrt{n}}, \ldots, a_{\sqrt{n}, \sqrt{n}], \text { where }}\right. \\
a_{p, r} & =\frac{1}{\sqrt{n}} e^{j 2 \pi\left[(p-1) \cos (\theta) \Delta_{v}+(r-1) \sin (\theta) \sin (\phi) \Delta_{h}\right]},
\end{aligned}
$$

while $\mathbf{w} \in \mathbb{C}^{n}$ is the beamforming vector that weighs the antenna array elements to steer the beam in the desired direction, and is represented by

$$
\begin{aligned}
\mathbf{w}\left(\theta_{s}, \phi_{s}\right) & =\left[w_{1,1}, w_{1,2}, \ldots, w_{1, \sqrt{n}}, \ldots, w_{\sqrt{n}, \sqrt{n}], \text { where }}\right. \\
w_{p, r} & =e^{-j 2 \pi\left[(p-1) \cos \left(\theta_{s}\right) \Delta_{v}+(r-1) \sin \left(\theta_{s}\right) \sin \left(\phi_{s}\right) \Delta_{h}\right]} .
\end{aligned}
$$

The factors $\Delta_{v}$ and $\Delta_{h}$ are the vertical and horizontal antenna spacings normalized to the wavelength, and are both set to 0.5 . We refer to [33] for a discussion on the patterns that can be generated for different combinations of steering angle and directions.

In our implementation, we associate a beamforming vector to each endpoint of each pair of connected devices (i.e., a base station and the user connected to it), and we update it by setting the steering angles equal to those corresponding to the LOS direction with a certain periodicity $T$, which is a parameter that can be set in the simulation scenario. By default, $T=20 \mathrm{~ms}$, one of the periodicities considered for the beamforming update in 3GPP NR [34]. Then, the beamforming gain $G_{i, j}$ is given (in $\mathrm{dB}$ ) by $A_{A, i}\left(\theta^{i, j}, \phi^{i, j}, \theta_{s}^{i}, \phi_{s}^{i}\right)+A_{A, j}\left(\theta^{j, i}, \phi^{j, i}, \theta_{s}^{j}, \phi_{s}^{j}\right)$. The gain is maximum only if the devices are connected, i.e., if $i$ is transmitting to $j$ or vice versa, and the steering angles match the direction between $i$ and $j$.

This beamforming model strikes a balance between complexity and flexibility. If the full channel matrix $\mathbf{H}$ is available in the simulation, as when using a SCM, then it is possible to model more realistically the beamforming gain, or, for example, compute the optimal beamforming vectors [14]. However, the complexity involved with SCM is much higher, as we will discuss in the next section.

\section{Channel Model Comparison}

In this section, we consider two different simulation scenarios, with different end-to-end transport protocols. In the first, 


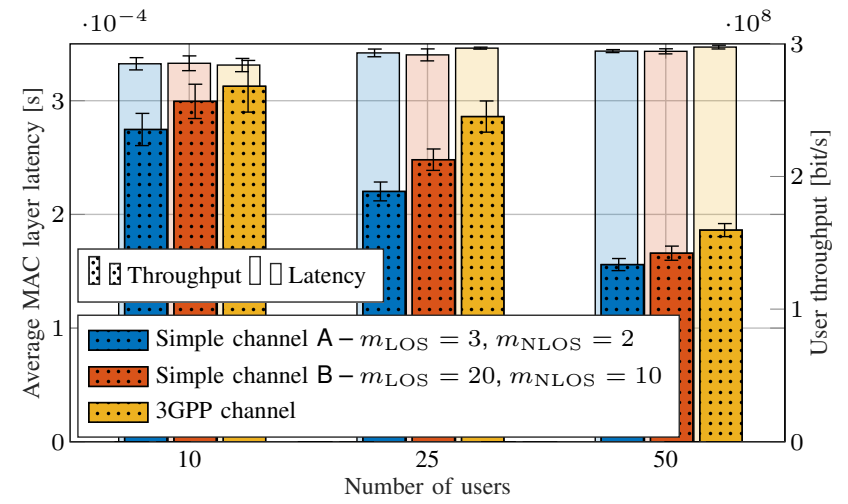

Figure 1: Average user throughput and latency for different channel models and number of users, in the scenario with UDP as transport.

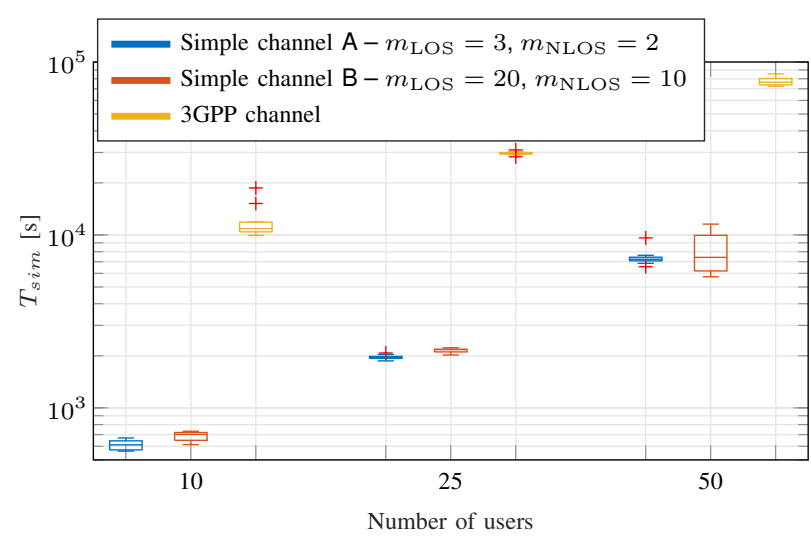

Figure 2: Boxplot for the simulation execution time, for different channel models and number of users, in the scenario with UDP as transport.

we deploy five base stations, in the center and at the four vertices of a square of side $200 \mathrm{~m}$. $N_{\mathrm{UE}} \in\{2,5,10\}$ users are randomly placed in a disc around each base station, for a total of 10,25 or 50 users. The base stations use a round robin scheduler. UDP is used as transport protocol to access data in a remote server, at a maximum rate of $400 \mathrm{Mbit} / \mathrm{s}$ per user. For the 3 GPP channel model, the selected scenario is Urban Macro. The results are averaged over 20 independent runs, each with a simulated time of $10 \mathrm{~s}$.

We compare the average user throughput and latency in Fig. 1 for different channel models. We test the 3GPP channel model and different $m$ values for the Nakagami fading in the simple channel model, i.e., setting $\mathrm{A}$ with $m_{\mathrm{LOS}}=3$, $m_{\mathrm{NLOS}}=2[23]$ and setting B with $m_{\mathrm{LOS}}=20, m_{\mathrm{NLOS}}=$ 10 [35], to simulate different impacts of the fading on the received signal. As it can be seen, there is no significant difference on the average MAC layer latency, which is in line with the results in [31]. For the throughput, instead, the simple channel model always shows an average throughput smaller than that of the 3GPP model. Therefore, the Nakagami fading-based channel represents in the simulated scenarios a conservative bound with respect to the measurement-based 3GPP channel. In particular, it can be seen that the throughput decreases as the severity of the fading phenomena increases. For the simple channel the throughput loss ranges from $13 \%$

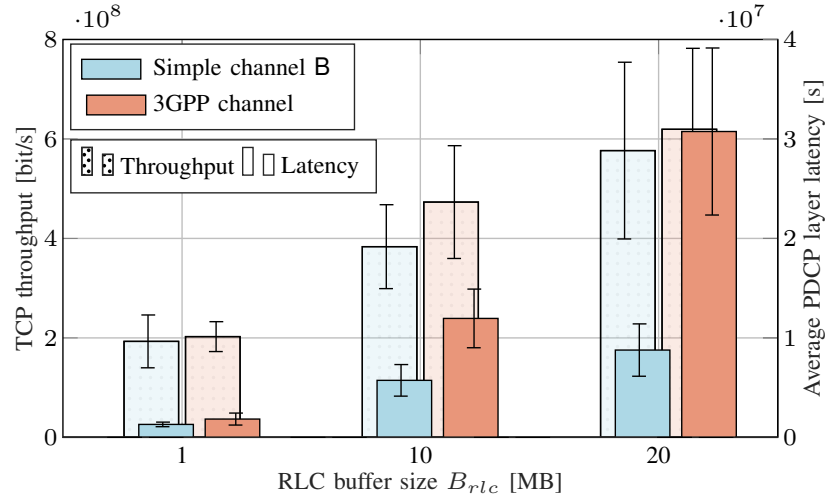

Figure 3: Average user throughput and latency for different channel models and Radio Link Control (RLC) buffer size $B_{r l c}$, in the scenario with TCP as transport.

(for $10 \mathrm{UEs}$ ) to $23 \%$ (for $25 \mathrm{UEs}$ ) with setting A, and from $4 \%$ (for $10 \mathrm{UEs}$ ) to $13 \%$ (for $25 \mathrm{UEs}$ ) with the less conservative setting B. Therefore, the simple channel model described in Sec. III is less accurate than the reference 3GPP model, given that it introduces more severe fading, even though it exhibits the same trend when varying the number of users in the simulation. However, as shown in Fig. 2, its usage reduces the simulation execution time $T_{\text {sim }}$ by an order of magnitude: for example, with 50 UEs, $T_{\text {sim }}$ is 123 minutes and 33 seconds with the simple channel with configuration $A$, and 1183 minutes and 30 seconds with the 3GPP channe 3 .

The second scenario, instead, involves a single user, three mmWave and one LTE base stations deployed as in [36]. The user moves in the scenario along a straight line for 100 $\mathrm{m}$, and hands over between the different base stations. TCP NewReno is used as transport protocol, and the results for the throughput and latency with different RLC buffer sizes $B_{\text {rlc }} \in\{1,10,20\} \mathrm{MB}$ and channel models (the 3GPP and the simple channel model with setting B) are shown in Fig 3 . In general, the 3GPP channel model has higher throughput and latency with respect to the simple model, even though for a small buffer size $B_{r l c}=1 \mathrm{MB}$ the performance is similar. For both channel models latency and throughput increase with the buffer size, as expected [36], but the latency increase is higher with the 3GPP model. This is due to TCP's behavior during the slow start phase, in which the number of bytes that are sent doubles at each Round Trip Time (RTT), until a packet is lost or the slow start threshold is reached. In these simulations, the latter is set to the maximum possible value of the TCP congestion window, thus TCP exits the slow start phase only because of packet loss. The 3GPP channel model incurs less severe fading than the simple channel model, therefore the probability of losing a packet because of random channel quality drops is smaller, and the slow start continues until the packet loss is triggered by an RLC buffer overflow. The consequence is that there is a latency spike due to excessive buffering at the RLC layer, which increases with the buffer size. On the other hand, with the simple channel model, TCP may exit earlier from slow start, and avoid overflowing the buffer

\footnotetext{
${ }^{3}$ All the simulations were run on a system with an Intel Xeon E5-2670v2 $2.5 \mathrm{GHz} \mathrm{CPU}$ and $40 \mathrm{~GB}$ of RAM.
} 
and causing the initial latency spike, at the price of a reduced throughput.

\section{COnCLUSIONS}

In this paper, we studied the impact of different channel models on end-to-end metrics and simulation complexity. In particular, we used the ns- 3 mmWave module, and the already implemented 3GPP channel model as a reference, and proposed the implementation of a simpler channel model, similar to those used for the mathematical analysis of mmWave networks, based on Nakagami fading and the computation of the beamforming gain from the array radiation pattern.

We showed that, with respect to the reference 3GPP model, the Nakagami fading-based model yields a lower throughput in end-to-end simulations with randomly generated scenarios, and both TCP and UDP as transport protocols. Moreover, when considering TCP, the different behavior and severity of the fading model generates different latency results, especially when larger buffer sizes in the base stations are considered. However, by using the simpler model it is possible to reduce the simulation execution time by an order of magnitude.

We believe that the insights we provided in this paper can provide guidance on which channel model should be used in mmWave simulations. When complex interactions between the channel and the end-to-end transport protocols are expected, it is better to use the reference 3GPP channel model, at the price of higher complexity. On the other hand, when the interplay between the transport and the lower layers is less complicated, a simpler model can be used to scale the simulations to a larger number of users, keeping in mind that the simulated throughput could be a lower bound with respect to that generated with the 3GPP channel.

As future work, we plan to develop a statistical model that can better abstract the behavior of the 3GPP model while reducing the simulation complexity, and continue to investigate which are the main accuracy trade offs with respect to the $3 \mathrm{GPP}$ channel model.

\section{REFERENCES}

[1] A. Osseiran, F. Boccardi, V. Braun, K. Kusume, P. Marsch, M. Maternia, O. Queseth, M. Schellmann, H. Schotten, H. Taoka et al., "Scenarios for 5G mobile and wireless communications: the vision of the METIS project," IEEE Commun. Mag., vol. 52, no. 5, pp. 26-35, May 2014.

[2] F. Boccardi, R. W. Heath Jr, A. Lozano, T. L. Marzetta, and P. Popovski, "Five disruptive technology directions for 5G," IEEE Commun. Mag., vol. 52, no. 2, pp. 74-80, Feb 2014.

[3] Z. Pi and F. Khan, "An introduction to millimeter-wave mobile broadband systems," IEEE Commun. Mag., vol. 49, no. 6, pp. 101-107, June 2011.

[4] S. Singh, F. Ziliotto, U. Madhow, E. M. Belding, and M. J. W. Rodwell, "Millimeter Wave WPAN: Cross-Layer Modeling and MultiHop Architecture," in 26th IEEE International Conference on Computer Communications (INFOCOM), May 2007, pp. 2336-2340.

[5] S. Sun, T. Rappaport, R. Heath, A. Nix, and S. Rangan, "MIMO for millimeter-wave wireless communications: beamforming, spatial multiplexing, or both?" IEEE Comm. Mag., vol. 52, no. 12, pp. 110-121, Dec. 2014.

[6] M. Akdeniz, Y. Liu, M. Samimi, S. Sun, S. Rangan, T. Rappaport, and E. Erkip, "Millimeter wave channel modeling and cellular capacity evaluation," IEEE J. Sel. Areas Commun., vol. 32, no. 6, pp. 1164-1179, June 2014.

[7] M. Zhang, M. Mezzavilla, R. Ford, S. Rangan, S. Panwar, E. Mellios, D. Kong, A. Nix, and M. Zorzi, "Transport layer performance in 5G mmWave cellular," in IEEE Conference on Computer Communications Workshops (INFOCOM WKSHPS), Apr 2016, pp. 730-735.
[8] M. Polese, R. Jana, and M. Zorzi, "TCP and MP-TCP in 5G mmWave Networks," IEEE Internet Comput., vol. 21, no. 5, pp. 12-19, Sep 2017.

[9] M. Polese, M. Giordani, M. Mezzavilla, S. Rangan, and M. Zorzi, "Improved Handover Through Dual Connectivity in 5G mmWave Mobile Networks," IEEE J. Sel. Areas Commun., vol. 35, no. 9, pp. 2069-2084, Sep 2017.

[10] P. Ferrand, M. Amara, S. Valentin, and M. Guillaud, "Trends and challenges in wireless channel modeling for evolving radio access," IEEE Commun. Mag., vol. 54, no. 7, pp. 93-99, July 2016.

[11] K. Brueninghaus, D. Astely, T. Salzer, S. Visuri, A. Alexiou, S. Karger, and G. A. Seraji, "Link performance models for system level simulations of broadband radio access systems," in IEEE 16th International Symposium on Personal, Indoor and Mobile Radio Communications, vol. 4, Sep 2005, pp. 2306-2311 Vol. 4.

[12] M. Mezzavilla, M. Zhang, M. Polese, R. Ford, S. Dutta, S. Rangan, and M. Zorzi, "End-to-End Simulation of $5 \mathrm{G}$ mmWave Networks," IEEE Commun. Surveys Tuts., April 2018.

[13] 3GPP, "Study on channel model for frequency spectrum above $6 \mathrm{GHz}$," TR 38.900 (Rel. 14), 2017.

[14] M. Zhang, M. Polese, M. Mezzavilla, S. Rangan, and M. Zorzi, "ns3 Implementation of the 3GPP MIMO Channel Model for Frequency Spectrum Above $6 \mathrm{GHz}$," in Proc. Workshop ns-3. ACM, 2017, pp. $71-78$.

[15] J. G. Andrews, T. Bai, M. N. Kulkarni, A. Alkhateeb, A. K. Gupta, and R. W. Heath, "Modeling and analyzing millimeter wave cellular systems," IEEE Trans. Commun., vol. 65, no. 1, pp. 403-430, Jan 2017.

[16] T. S. Rappaport, F. Gutierrez, E. Ben-Dor, J. N. Murdock, Y. Qiao, and J. I. Tamir, "Broadband millimeter-wave propagation measurements and models using adaptive-beam antennas for outdoor urban cellular communications," IEEE Trans. Antennas Propag., vol. 61, no. 4, pp. 1850-1859, Apr 2013.

[17] R. J. Weiler, M. Peter, W. Keusgen, H. Shimodaira, K. T. Gia, and K. Sakaguchi, "Outdoor millimeter-wave access for heterogeneous networks path loss and system performance," in IEEE 25th Annual International Symposium on Personal, Indoor, and Mobile Radio Communication (PIMRC), Sep 2014, pp. 2189-2193.

[18] I. Hemadeh, K. Satyanarayana, M. El-Hajjar, and L. Hanzo, "Millimeterwave communications: Physical channel models, design considerations, antenna constructions and link-budget," IEEE Commun. Surveys Tuts., 2018.

[19] T. S. Rappaport, Y. Xing, G. R. MacCartney, A. F. Molisch, E. Mellios, and J. Zhang, "Overview of Millimeter Wave Communications for FifthGeneration (5G) Wireless Networks - With a Focus on Propagation Models," IEEE Trans. Antennas Propag., vol. 65, no. 12, pp. 6213-6230, Dec 2017.

[20] METIS2020, Deliverable, "D1.4 v3, METIS Channel Model," 2015.

[21] S. Jaeckel, L. Raschkowski, K. Borner, and L. Thiele, "QuaDRiGa: A 3-D Multi-Cell Channel Model With Time Evolution for Enabling Virtual Field Trials," IEEE Trans. Antennas Propag., vol. 62, no. 6, pp. 32423256, Mar 2014.

[22] IST WINNER II, "D1.1.2 V1.2 WINNER II Channel Models," 2007.

[23] T. Bai and R. W. Heath, "Coverage and rate analysis for millimeter-wave cellular networks," IEEE Trans. Wireless Commun., vol. 14, no. 2, pp. 1100-1114, Feb 2015.

[24] G. R. MacCartney, T. S. Rappaport, and S. Rangan, "Rapid Fading Due to Human Blockage in Pedestrian Crowds at 5G Millimeter-Wave Frequencies," in Proc. IEEE GLOBECOM, Dec 2017.

[25] M. Nakagami, "The m-distribution-a general formula of intensity distribution of rapid fading," in Statistical Methods in Radio Wave Propagation. Pergamon, 1960, pp. 3 - 36.

[26] S. Singh, M. N. Kulkarni, A. Ghosh, and J. G. Andrews, "Tractable model for rate in self-backhauled millimeter wave cellular networks," IEEE $J$. Sel. Areas Commun., vol. 33, no. 10, pp. 2196-2211, Oct 2015.

[27] A. Alkhateeb, Y. H. Nam, M. S. Rahman, J. Zhang, and R. W. Heath, "Initial beam association in millimeter wave cellular systems: Analysis and design insights," IEEE Trans. Wireless Commun., vol. 16, no. 5, pp. 2807-2821, May 2017.

[28] J. Park, S. L. Kim, and J. Zander, "Tractable resource management with uplink decoupled millimeter-wave overlay in ultra-dense cellular networks," IEEE Trans. Wireless Commun., vol. 15, no. 6, pp. 43624379, June 2016.

[29] C. T. Neil, M. Shafi, P. J. Smith, P. A. Dmochowski, and J. Zhang, "Impact of Microwave and mmWave Channel Models on 5G Systems Performance," IEEE Trans. Antennas Propag., vol. 65, no. 12, pp. 65056520, Dec 2017. 
[30] K. Zeman, P. Masek, M. Stusek, J. Hosek, and P. Silhavy, "Accuracy comparison of propagation models for mmwave communication in ns-3," in 9th International Congress on Ultra Modern Telecommunications and Control Systems and Workshops (ICUMT), Nov 2017, pp. 334-340.

[31] S. Dutta, M. Mezzavilla, R. Ford, M. Zhang, S. Rangan, and M. Zorzi, "Frame structure design and analysis for millimeter wave cellular systems," IEEE Trans. Wireless Commun., vol. 16, no. 3, pp. 1508-1522, Mar 2017.

[32] 3GPP, "Study of Radio Frequency (RF) and Electromagnetic Compatibility (EMC) requirements for Active Antenna Array System (AAS) base station," TR 37.840 (12.1.0), 2013.

[33] M. Rebato, L. Resteghini, C. Mazzucco, and M. Zorzi, "Study of Realistic Antenna Patterns in 5G mmWave Cellular Scenarios," in IEEE International Conference on Communications (ICC), May 2018. [Online]. Available: https://arxiv.org/abs/1802.01316

[34] 3GPP, "Discussion on remaining issues of SS block and SS burst set," Motorola Mobility, Lenovo - Tdoc R1-1714212, 2017.

[35] A. K. Gupta, J. G. Andrews, and R. W. Heath, "On the feasibility of sharing spectrum licenses in mmwave cellular systems," IEEE Trans. Commun., vol. 64, no. 9, pp. 3981-3995, Sep 2016.

[36] M. Polese, M. Mezzavilla, S. Rangan, and M. Zorzi, "Mobility Management for TCP in mmWave Networks," in Proc. 1st ACM Workshop on Millimeter-Wave Networks and Sensing Systems, ser. mmNets '17, 2017, pp. 11-16. 